\title{
Partial derivatives of some types of two-variables functions
}

\author{
Chii-Huei Yu \\ Department of Management and Information, Nan Jeon Institute of Technology, Tainan City, Taiwan
}

Email address:

chiihuei@mail.njtc.edu.tw (Chii-Huei Yu)

\section{To cite this article:}

Chii-Huei Yu. Partial Derivatives of Some Types of Two-Variables Functions. Pure and Applied Mathematics Journal. Vol. 2, No. 2, 2013, pp. 56-61. doi: 10.11648/j.pamj.20130202.12

\begin{abstract}
This paper mainly studies the evaluation of partial derivatives of four types of two-variables functions. We can obtain the infinite series forms of any order partial derivatives of these four types of functions by using differentiation term by term theorem, and hence reducing the difficulty of calculating their higher order partial derivative values greatly. On the other hand, we propose four functions of two-variables to evaluate their any order partial derivatives, and some of their higher order partial derivative values practically. At the same time, we employ Maple to calculate the approximations of these higher order partial derivative values and their infinite series forms for verifying our answers.
\end{abstract}

Keywords: Partial Derivatives, Differentiation Term By Term Theorem, Infinite Series Forms, Maple

\section{Introduction}

In calculus and engineering mathematics courses, the research about the partial derivatives of multivariable functions is an important issue. For example, Laplace equation, wave equation, as well as some other important physical equations are involved the partial derivatives of multivariable functions. Therefore, whether in physics, engineering or other sciences, the evaluation and numerical calculation of the partial derivatives has its importance. Books and papers in this regard can refer to [1-9]. In this paper, we mainly study the evaluation of partial derivatives of the following four types of two-variables functions

$$
\begin{array}{r}
f_{1}(x, y) \\
=\cos [m(c x+d)] \cdot \sin \left[y^{r} \cos (c x+d)\right] \cdot \cosh \left[y^{r} \sin (c x+d)\right] \\
-\sin [m(c x+d)] \cdot \cos \left[y^{r} \cos (c x+d)\right] \cdot \sinh \left[y^{r} \sin (c x+d)\right](1) \\
f_{2}(x, y) \\
+\sin [m(c x+d)] \cdot \sin \left[y^{r} \cos (c x+d)\right] \cdot \cosh \left[y^{r} \sin (c x+d)\right](2)
\end{array}
$$

$$
\begin{array}{r}
g_{1}(x, y) \\
=\cos [m(c x+d)] \cdot \cos \left[y^{r} \cos (c x+d)\right] \cdot \cosh \left[y^{r} \sin (c x+d)\right] \\
+\sin [m(c x+d)] \cdot \sin \left[y^{r} \cos (c x+d)\right] \cdot \sinh \left[y^{r} \sin (c x+d)\right](3) \\
=\sin [m(c x+d)] \cdot \cos \left[y^{r} \cos (c x+d)\right] \cdot \cosh \left[y^{r} \sin (c x+d)\right] \\
-\cos [m(c x+d)] \cdot \sin \left[y^{r} \cos (c x+d)\right] \cdot \sinh \left[y^{r} \sin (c x+d)\right](4)
\end{array}
$$

where $r, c, d$ are real numbers, and $m$ is any integer. We can obtain the infinite series forms of these four types of functions by using differentiation term by term theorem, that is, the major results in this paper: Theorems 1,2,3, and 4. On the other hand, we propose four functions of two-variables to evaluate their any order partial derivatives, and some of their higher order partial derivative values practically. Simultaneously, we employ Maple to calculate the approximations of these higher order partial derivative values and their infinite series forms for verifying our answers.

\section{Main Results}

Firstly, we introduce some notations, formulas and an important theorem used in this paper.

Notations: 
(i) Suppose that $t$ is any real number, and $m$ is any positive integer. Define $(t)_{m}=t(t-1) \cdots(t-m+1)$, and $(t)_{0}=1$.

(ii) Assume that $q, p$ are non-negative integers. For the two-variables function $f(x, y)$, the $p$-times partial derivative with respect to $x$, and then $q$-times partial derivative with respect to $y$ is a $(q+p)$-th order partial derivative of $f(x, y)$, and denoted by $\frac{\partial^{q+p} f}{\partial y^{q} \partial x^{p}}(x, y)$.

Formulas:

In the following, we assume that $\theta, a, b$ are arbitrary real numbers, $z$ is any non-zero complex number, $m$ is any integer, and $i=\sqrt{-1}$.

(i) Euler's formula:

$$
e^{i \theta}=\cos \theta+i \sin \theta
$$

(ii) DeMoivre's formula :

$$
(\cos \theta+i \sin \theta)^{m}=\cos m \theta+i \sin m \theta .
$$

(iii) $z^{m} \sin z=\sum_{n=1}^{\infty} \frac{(-1)^{n-1}}{(2 n-1) !} z^{2 n+m-1}$.

(iv) $z^{m} \cos z=\sum_{n=0}^{\infty} \frac{(-1)^{n}}{(2 n) !} z^{2 n+m}$.

(v) $\sin (a+i b)=\sin a \cdot \cosh b+i \cos a \cdot \sinh b$.

(vi) $\cos (a+i b)=\cos a \cdot \cosh b-i \sin a \cdot \sinh b$.

Differentiation term by term theorem ([10]).

If, for all non-negative integer $k$, the functions

$g_{k}:(a, b) \rightarrow R$ satisfy the following three conditions : (i) there exists a point $x_{0} \in(a, b)$ such that $\sum_{k=0}^{\infty} g_{k}\left(x_{0}\right)$ is convergent, (ii) all functions $g_{k}(x)$ are differentiable on open interval $(a, b)$, (iii) $\sum_{k=0}^{\infty} \frac{d}{d x} g_{k}(x)$ is uniformly convergent on $(a, b)$. Then $\sum_{k=0}^{\infty} g_{k}(x)$ is uniformly convergent and differentiable on $(a, b)$. Moreover, its derivative $\frac{d}{d x} \sum_{k=0}^{\infty} g_{k}(x)=\sum_{k=0}^{\infty} \frac{d}{d x} g_{k}(x)$.

Next, we derive the first result in this article.

Theorem 1. Assume that $r, c, d$ are real numbers, $m$ is any integer, $q, p$ are any non-negative integers, and suppose that the domain of the two-variables function

$$
f_{1}(x, y)
$$

$=\cos [m(c x+d)] \cdot \sin \left[y^{r} \cos (c x+d)\right] \cdot \cosh \left[y^{r} \sin (c x+d)\right]$

$-\sin [m(c x+d)] \cdot \cos \left[y^{r} \cos (c x+d)\right] \cdot \sinh \left[y^{r} \sin (c x+d)\right]$

is $\left\{(x, y) \in R^{2} \mid y^{r}\right.$ exist,$\left.y \neq 0\right\}$. Then the $(q+p)$-th order par- tial derivative of $f_{1}(x, y)$,

$$
\begin{gathered}
\frac{\partial^{q+p} f_{1}}{\partial y^{q} \partial x^{p}}(x, y) \\
=c^{p} \cdot \sum_{n=1}^{\infty} \frac{(-1)^{n-1}(2 n r-r)_{q} \cdot(2 n+m-1)^{p}}{(2 n-1) !} \cdot y^{2 n r-r-q} . \\
\cos \left[(2 n+m-1)(c x+d)+\frac{p \pi}{2}\right]
\end{gathered}
$$

Proof. Let $z=y^{r} \exp [i(c x+d)]$, then by formula (iii), Euler's formula and DeMoivre's formula, we obtain

$$
\begin{aligned}
& y^{m r} \exp [i m(c x+d)] \cdot \sin \left\{y^{r} \exp [i(c x+d)]\right\} \\
= & \sum_{n=1}^{\infty} \frac{(-1)^{n-1}}{(2 n-1) !} \cdot y^{(2 n+m-1) r} \cdot \exp [i(2 n+m-1)(c x+d)]
\end{aligned}
$$

, and hence

$$
\begin{gathered}
{[\cos m(c x+d)+i \sin m(c x+d)] \times} \\
\sin \left[y^{r} \cos (c x+d)+i y^{r} \sin (c x+d)\right] \\
=\sum_{n=1}^{\infty} \frac{(-1)^{n-1}}{(2 n-1) !} \cdot y^{(2 n-1) r}\{\cos [(2 n+m-1)(c x+d)+ \\
i \sin [(2 n+m-1)(c x+d)]\}
\end{gathered}
$$

Using formula (v) and the equality of the real part of both sides of (7), we have

$$
\begin{aligned}
& \cos [m(c x+d)] \cdot \sin \left[y^{r} \cos (c x+d)\right] \cdot \cosh \left[y^{r} \sin (c x+d)\right] \\
& -\sin [m(c x+d)] \cdot \cos \left[y^{r} \cos (c x+d)\right] \cdot \sinh \left[y^{r} \sin (c x+d)\right] \\
& =\sum_{n=1}^{\infty} \frac{(-1)^{n-1}}{(2 n-1) !} \cdot y^{2 n r-r} \cos [(2 n+m-1)(c x+d)]
\end{aligned}
$$

Therefore, by differentiation term by term theorem, we obtain the $(q+p)$-th order partial derivative of $f_{1}(x, y)$,

$$
\begin{gathered}
\frac{\partial^{q+p} f_{1}}{\partial y^{q} \partial x^{p}}(x, y) \\
=c^{p} \cdot \sum_{n=1}^{\infty} \frac{(-1)^{n-1}(2 n r-r)_{q} \cdot(2 n+m-1)^{p}}{(2 n-1) !} \cdot y^{2 n r-r-q} . \\
\cos \left[(2 n+m-1)(c x+d)+\frac{p \pi}{2}\right]
\end{gathered}
$$

Using the equality of the imaginary part of both sides of (7), and the same proof as Theorem 1, we immediately 
have the following second result in this paper.

Theorem 2. The assumptions are the same as Theorem 1, and the domain of the two-variables function

$$
f_{2}(x, y)
$$

$=\cos [m(c x+d)] \cdot \cos \left[y^{r} \cos (c x+d)\right] \cdot \sinh \left[y^{r} \sin (c x+d)\right]$

$+\sin [m(c x+d)] \cdot \sin \left[y^{r} \cos (c x+d)\right] \cdot \cosh \left[y^{r} \sin (c x+d)\right]$

is $\left\{(x, y) \in R^{2} \mid y^{r}\right.$ exist, $\left.y \neq 0\right\}$. Then the $(q+p)$-th order partial derivative of $f_{2}(x, y)$,

$$
\begin{gathered}
\frac{\partial^{q+p} f_{2}}{\partial y^{q} \partial x^{p}}(x, y) \\
=c^{p} \cdot \sum_{n=1}^{\infty} \frac{(-1)^{n-1}(2 n r-r)_{q} \cdot(2 n+m-1)^{p}}{(2 n-1) !} \cdot y^{2 n r-r-q} . \\
\sin \left[(2 n+m-1)(c x+d)+\frac{p \pi}{2}\right]
\end{gathered}
$$

Below we derive the third result in this article.

Theorem 3. The same assumptions as Theorem 1, and the domain of

$$
g_{1}(x, y)
$$

$=\cos [m(c x+d)] \cdot \cos \left[y^{r} \cos (c x+d)\right] \cdot \cosh \left[y^{r} \sin (c x+d)\right]$ $+\sin [m(c x+d)] \cdot \sin \left[y^{r} \cos (c x+d)\right] \cdot \sinh \left[y^{r} \sin (c x+d)\right]$

is $\left\{(x, y) \in R^{2} \mid y^{r}\right.$ exist, $\left.y \neq 0\right\}$. Then the $(q+p)$-th order partial derivative of $g_{1}(x, y)$,

$$
\begin{aligned}
& \frac{\partial^{q+p} g_{1}}{\partial y^{q} \partial x^{p}}(x, y)=c^{p} \cdot \sum_{n=0}^{\infty} \frac{(-1)^{n}(2 n r)_{q} \cdot(2 n+m)^{p}}{(2 n) !} \cdot y^{2 n r-q} \\
& \cos \left[(2 n+m)(c x+d)+\frac{p \pi}{2}\right]
\end{aligned}
$$

Proof. Also, we let $z=y^{r} \exp [i(c x+d)]$, then by formula (iv), Euler's formula and DeMoivre's formula, we obtain

$$
\begin{array}{r}
y^{m r} \exp [\operatorname{im}(c x+d)] \cdot \cos \left\{y^{r} \exp [i(c x+d)]\right\} \\
=\sum_{n=0}^{\infty} \frac{(-1)^{n}}{(2 n) !} \cdot y^{(2 n+m) r} \cdot \exp [i(2 n+m)(c x+d)]
\end{array}
$$

Therefore,

$$
[\cos m(c x+d)+i \sin m(c x+d)] \times
$$
$\cos \left[y^{r} \cos (c x+d)+i y^{r} \sin (c x+d)\right]=$

$$
\left.\sum_{n=0}^{\infty} \frac{(-1)^{n}}{(2 n) !} \cdot y^{2 n r}\{\cos [2 n+m)(c x+d)]+i \sin [2 n+m)(c x+d)\right]
$$

By formula (vi) and the equality of the real part of both sides of (12), we have

$\cos [m(c x+d)] \cdot \cos \left[y^{r} \cos (c x+d)\right] \cdot \cosh \left[y^{r} \sin (c x+d)\right]$

$+\sin [m(c x+d)] \cdot \sin \left[y^{r} \cos (c x+d)\right] \cdot \sinh \left[y^{r} \sin (c x+d)\right]$

$$
=\sum_{n=0}^{\infty} \frac{(-1)^{n}}{(2 n) !} \cdot y^{2 n r} \cos [(2 n+m)(c x+d)]
$$

Thus, by differentiation term by term theorem, we obtain the $(q+p)$-th order partial derivative of $g_{1}(x, y)$,

$$
\begin{gathered}
\frac{\partial^{q+p} g_{1}}{\partial y^{q} \partial x^{p}}(x, y)=c^{p} \cdot \sum_{n=0}^{\infty} \frac{(-1)^{n}(2 n r)_{q} \cdot(2 n+m)^{p}}{(2 n) !} \cdot y^{2 n r-q} . \\
\cos \left[(2 n+m)(c x+d)+\frac{p \pi}{2}\right]
\end{gathered}
$$

From the equality of the imaginary part of both sides of (12), and use the same proof as Theorem 3, we immediately obtain the fourth result in this paper.

Theorem 4. The same assumptions as Theorem 1, and the domain of

$$
g_{2}(x, y)
$$

$=\sin [m(c x+d)] \cdot \cos \left[y^{r} \cos (c x+d)\right] \cdot \cosh \left[y^{r} \sin (c x+d)\right]$

$-\cos [m(c x+d)] \cdot \sin \left[y^{r} \cos (c x+d)\right] \cdot \sinh \left[y^{r} \sin (c x+d)\right]$

is $\left\{(x, y) \in R^{2} \mid y^{r}\right.$ exist, $\left.y \neq 0\right\}$. Then the $(q+p)$-th order partial derivative of $g_{2}(x, y)$,

$$
\begin{aligned}
\frac{\partial^{q+p} g_{2}}{\partial y^{q} \partial x^{p}}(x, y)= & c^{p} \cdot \sum_{n=0}^{\infty} \frac{(-1)^{n}(2 n r)_{q} \cdot(2 n+m)^{p}}{(2 n) !} \cdot y^{2 n r-q} . \\
& \sin \left[(2 n+m)(c x+d)+\frac{p \pi}{2}\right]
\end{aligned}
$$

\section{Examples}

In the following, we propose four functions of two- variables to find their any order partial derivatives, and some of their higher order partial derivative values practically. On the other hand, we use Maple to calculate the approximations of these higher-order partial derivative values and their infinite series forms.

Example 1. Suppose that the domain of the following two-variables function 


$$
f_{1}(x, y)=
$$

$\cos [4(2 x-5)] \cdot \sin \left[y^{3} \cos (2 x-5)\right] \cdot \cosh \left[y^{3} \sin (2 x-5)\right]$

$-\sin [4(2 x-5)] \cdot \cos \left[y^{3} \cos (2 x-5)\right] \cdot \sinh \left[y^{3} \sin (2 x-5)\right]$

is $\left\{(x, y) \in R^{2} \mid y \neq 0\right\}$. By Theorem 1, we obtain the infi-

nite series forms of any $(q+p)$-th order partial derivatives of $f_{1}(x, y)$ as follows.

$$
\begin{aligned}
& \frac{\partial^{q+p} f_{1}}{\partial y^{q} \partial x^{p}}(x, y)=2^{p} \cdot \sum_{n=1}^{\infty} \frac{(-1)^{n-1}(6 n-3)_{q} \cdot(2 n+3)^{p}}{(2 n-1) !} \cdot y^{6 n-3-q} \\
& \cos \left[(2 n+3)(2 x-5)+\frac{p \pi}{2}\right]
\end{aligned}
$$

Therefore, we have the 6-th order partial derivatives of $f_{1}(x, y)$ at $(3,2)$,

$$
=8 \cdot \sum_{n=1}^{\infty} \frac{(-1)^{n-1}(6 n-3)_{3} \cdot(2 n+3)^{3}}{(2 n-1) !} \cdot 2^{6 n-6} \cdot \sin (2 n+3)(16
$$

Next, we use Maple to verify our answer.

$$
\begin{gathered}
>\mathrm{f} 1:=(\mathrm{x}, \mathrm{y})->\cos (4 *(2 * \mathrm{x}-5)) * \sin \left(\mathrm{y}^{\wedge} 3 * \cos (2 * \mathrm{x}-5)\right) * \cosh \left(\mathrm{y}^{\wedge} 3\right. \\
\left.\sin ^{*}(2 * \mathrm{x}-5)\right)-\sin (4 *(2 * \mathrm{x}-5)) * \cos \left(\mathrm{y}^{\wedge} 3 * \cos (2 * \mathrm{x}-5)\right) * \sinh \left(\mathrm{y}^{\wedge} 3\right. \\
* \sin (2 * \mathrm{x}-5)) ; \\
f l:=(x, y) \rightarrow
\end{gathered}
$$

$\cos (8 x-20) \sin \left(y^{3} \cos (2 x-5)\right) \cosh \left(y^{3} \sin (2 x-5)\right)-$ $\sin (8 x-20) \cos \left(y^{3} \cos (2 x-5)\right) \sinh \left(y^{3} \sin (2 x-5)\right)$

$>\operatorname{evalf}(\mathrm{D}[1 \$ 3,2 \$ 3](\mathrm{f1})(3,2), 14)$;

$$
-2.2023727438581 \cdot 10^{10}
$$

$>\operatorname{evalf}\left(8 * \operatorname{sum}\left((-1)^{\wedge}(n-1)^{*} \operatorname{product}(6 * n-3-\mathrm{j}, \mathrm{j}=0 . .2) *(2 * n+3)^{\wedge}\right.\right.$

$3 /(2 * n-1) ! * 2 \wedge(6 * n-6) * \sin (2 * n+3), n=1$..infinity $), 14)$;

$$
-2.2023727438580 \cdot 10^{10}+3.2805000000000 \cdot 10^{-8} \mathrm{I}
$$

The above answer obtained by Maple appears the imaginary number $\mathrm{I}(=\sqrt{-1})$, that is because Maple uses its own built-in special functions to calculate it. But the imaginary part is very small, so can be ignored.

Example 2. Assume that the domain of

$$
\begin{gathered}
f_{2}(x, y) \\
=\cos [2(5 x+3)] \cdot \cos \left[y^{1 / 2} \cos (5 x+3)\right] \cdot \sinh \left[y^{1 / 2} \sin (5 x+3)\right] \\
+\sin [2(5 x+3)] \cdot \sin \left[y^{1 / 2} \cos (5 x+3)\right] \cdot \cosh \left[y^{1 / 2} \sin (5 x+3)\right]
\end{gathered}
$$

is $\left\{(x, y) \in R^{2} \mid y>0\right\}$. Using Theorem 2, we obtain the following infinite series forms of any $(q+p)$-th order partial derivatives of $f_{2}(x, y)$

$$
\begin{aligned}
\frac{\partial^{q+p} f_{2}}{\partial y^{q} \partial x^{p}}(x, y)= & 5^{p} \cdot \sum_{n=1}^{\infty} \frac{(-1)^{n-1}(n-1 / 2)_{q} \cdot(2 n+1)^{p}}{(2 n-1) !} \cdot y^{n-1 / 2-q} . \\
& \sin \left[(2 n+1)(5 x+3)+\frac{p \pi}{2}\right]
\end{aligned}
$$

So we can evaluate the following 7-th order partial derivatives of $f_{2}(x, y)$ at $(-1,4)$

$$
=5^{5} \cdot \sum_{n=1}^{\infty} \frac{(-1)^{n-1}(n-1 / 2)_{2} \cdot(2 n+1)^{5}}{(2 n-1) !} \cdot 4^{n-5 / 2} \cdot \cos (4 n+2)(18)
$$

Also, we use Maple to verify our answer.

$>\mathrm{f} 2:=(\mathrm{x}, \mathrm{y})->\cos \left(2 *\left(5^{*} \mathrm{x}+3\right)\right) * \cos \left(\mathrm{y}^{\wedge}(1 / 2) * \cos \left(5^{*} \mathrm{x}+3\right)\right) * \sinh$ $\left(\mathrm{y}^{\wedge}(1 / 2)^{*} \sin \left(5^{*} \mathrm{x}+3\right)\right)+\sin \left(2 *\left(5^{*} \mathrm{x}+3\right)\right) * \sin \left(\mathrm{y}^{\wedge}(1 / 2) * \cos \left(5^{*} \mathrm{x}\right.\right.$ $+3))^{*} \cosh \left(\mathrm{y}^{\wedge}(1 / 2) * \sin \left(5^{*} \mathrm{x}+3\right)\right)$;

$$
f 2:=(x, y) \rightarrow
$$

$\cos (10 x+6) \cos (\sqrt{y} \cos (5 x+3)) \sinh (\sqrt{y} \sin (5 x+3))+$ $\sin (10 x+6) \sin (\sqrt{y} \cos (5 x+3)) \cosh (\sqrt{y} \sin (5 x+3))$ $>\operatorname{evalf}(\mathrm{D}[1 \$ 5,2 \$ 2](\mathrm{f} 2)(-1,4), 14)$;

$$
-1.5112970990434 \cdot 10^{6}
$$

$>\operatorname{evalf}\left(5^{\wedge} 5^{*} \operatorname{sum}\left((-1)^{\wedge}(\mathrm{n}-1) * \operatorname{product}(\mathrm{n}-1 / 2-\mathrm{j}, \mathrm{j}=0 . .1) *(2 * \mathrm{n}+1\right.\right.$ )$^{\wedge} 5 /(2 * n-1) ! * 4 \wedge(n-5 / 2) * \cos (4 * n+2), n=1$..infinity $\left.), 14\right)$;

\section{$-1.5112970990436 \cdot 10^{6}+0 . \mathrm{I}$}

The above answer obtained by Maple also appears the imaginary number I, but the imaginary part is 0 , so can be ignored.

Example 3. If the domain of the two-variables function

$$
\begin{gathered}
g_{1}(x, y) \\
=\cos [7(4 x-1)] \cdot \cos \left[y^{2} \cos (4 x-1)\right] \cdot \cosh \left[y^{2} \sin (4 x-1)\right] \\
+\sin [7(4 x-1)] \cdot \sin \left[y^{2} \cos (4 x-1)\right] \cdot \sinh \left[y^{2} \sin (4 x-1)\right] \\
\text { is }\left\{(x, y) \in R^{2} \mid y \neq 0\right\} . \text { Then by Theorem 3, the infinite }
\end{gathered}
$$
series forms of any $(q+p)$-th order partial derivatives of $g_{1}(x, y)$ is 


$$
\begin{gathered}
\frac{\partial^{q+p} g_{1}}{\partial y^{q} \partial x^{p}(x, y)}=4^{p} \cdot \sum_{n=0}^{\infty} \frac{(-1)^{n}(4 n)_{q} \cdot(2 n+7)^{p}}{(2 n) !} \cdot y^{4 n-q} . \\
\cos \left[(2 n+7)(4 x-1)+\frac{p \pi}{2}\right]
\end{gathered}
$$

Thus, we obtain the following 5 -th order partial derivatives of $g_{1}(x, y)$ at $\left(\frac{1}{2}, 3\right)$

$$
\begin{array}{r}
\frac{\partial^{5} g_{1}}{\partial y^{3} \partial x^{2}}\left(\frac{1}{2}, 3\right) \\
=-16 \cdot \sum_{n=0}^{\infty} \frac{(-1)^{n}(4 n)_{3} \cdot(2 n+7)^{2}}{(2 n) !} \cdot 3^{4 n-3} \cdot \cos (2 n+7)(20)
\end{array}
$$

Using Maple to calculate the approximations of $\frac{\partial^{5} g_{1}}{\partial y^{3} \partial x^{2}}\left(\frac{1}{2}, 3\right)$ and its infinite series forms as follows.

$>\mathrm{g} 1:=(\mathrm{x}, \mathrm{y})->\cos \left(7^{*}\left(4^{*} \mathrm{x}-1\right)\right) * \cos \left(\mathrm{y}^{\wedge} 2 * \cos \left(4^{*} \mathrm{x}-1\right)\right) * \cosh \left(\mathrm{y}^{\wedge}\right.$ $2 * \sin (4 * x-1))+\sin (7 *(4 * x-1)) * \sin \left(y^{\wedge} 2 * \cos (4 * x-1)\right) * \sinh \left(y^{\wedge}\right.$ $2 * \sin (4 * x-1))$

$$
g 1:=(x, y) \rightarrow
$$

$\cos (28 x-7) \cos \left(y^{2} \cos (4 x-1)\right) \cosh \left(y^{2} \sin (4 x-1)\right)+$ $\sin (28 x-7) \sin \left(y^{2} \cos (4 x-1)\right) \sinh \left(y^{2} \sin (4 x-1)\right)$

$$
>\operatorname{evalf}(\mathrm{D}[1 \$ 2,2 \$ 3](\mathrm{g} 1)(1 / 2,3), 14) \text {; }
$$

$$
-1.2970725043722 \cdot 10^{9}
$$

$>\operatorname{evalf}\left(-16^{*} \operatorname{sum}\left((-1)^{\wedge} n * \operatorname{product}(4 * n-\mathrm{j}, \mathrm{j}=0 . .2)^{*}(2 * n+7)^{\wedge} 2 /(2\right.\right.$ $* n) ! * 3 \wedge(4 * n-3) * \cos (2 * n+7), n=0$.infinity $), 14)$;

$$
-1.2970725043722 \cdot 10^{9}+0 . \mathrm{I}
$$

The imaginary part of the above answer obtained by Maple is 0 , so can be ignored.

Example 4. Suppose that the domain of

$$
\begin{gathered}
g_{2}(x, y) \\
=\sin [5(6 x-7)] \cdot \cos \left[y^{4} \cos (6 x-7)\right] \cdot \cosh \left[y^{4} \sin (6 x-7)\right] \\
-\cos [5(6 x-7)] \cdot \sin \left[y^{4} \cos (6 x-7)\right] \cdot \sinh \left[y^{4} \sin (6 x-7)\right] \\
\text { is }\left\{(x, y) \in R^{2} \mid y \neq 0\right\} . \text { Using Theorem 4, the infinite se- }
\end{gathered}
$$
ries forms of any $(q+p)$-th order partial derivatives of $g_{2}(x, y)$ is

$$
\frac{\partial^{q+p} g_{2}}{\partial y^{q} \partial x^{p}}(x, y)=6^{p} \cdot \sum_{n=0}^{\infty} \frac{(-1)^{n}(8 n)_{q} \cdot(2 n+5)^{p}}{(2 n) !} \cdot y^{8 n-q} .
$$

$$
\sin \left[(2 n+5)(6 x-7)+\frac{p \pi}{2}\right]
$$

So we obtain the following 7 -th order partial derivatives of $g_{2}(x, y)$ at $(1,2)$

$$
\begin{array}{r}
\frac{\partial^{7} g_{2}}{\partial y^{3} \partial x^{4}}(1,2) \\
=-6^{4} \cdot \sum_{n=0}^{\infty} \frac{(-1)^{n}(8 n)_{3} \cdot(2 n+5)^{4}}{(2 n) !} \cdot 2^{8 n-3} \cdot \sin (2 n+5) \quad(22)
\end{array}
$$

Also, we use Maple to verify our answer.

$$
\begin{gathered}
>\mathrm{g} 2:=(\mathrm{x}, \mathrm{y})->\sin \left(5^{*}\left(6^{*} \mathrm{x}-7\right)\right) * \cos \left(\mathrm{y}^{\wedge} 4 * \cos \left(6^{*} \mathrm{x}-7\right)\right) * \cosh \left(\mathrm{y}^{\wedge} 4\right. \\
* \sin (6 * \mathrm{x}-7))-\cos \left(5^{*}\left(6^{*} \mathrm{x}-7\right)\right) * \sin \left(\mathrm{y}^{\wedge} 4 * \cos \left(6^{*} \mathrm{x}-7\right)\right) * \sinh \left(\mathrm{y}^{\wedge} 4\right. \\
\left.* \sin \left(6^{*} \mathrm{x}-7\right)\right) ; \\
\mathrm{g} 2:=(x, y) \rightarrow \\
\sin (30 x-35) \cos \left(y^{4} \cos (6 x-7)\right) \cosh \left(y^{4} \sin (6 x-7)\right)- \\
\cos (30 x-35) \sin \left(y^{4} \cos (6 x-7)\right) \sinh \left(y^{4} \sin (6 x-7)\right) \\
>\operatorname{evalf}(\mathrm{D}[1 \$ 4,2 \$ 3](\mathrm{g} 2)(1,2), 14) ; \\
2.7815111865636 \cdot 10^{18}
\end{gathered}
$$

$>\operatorname{evalf}\left(-6^{\wedge} 4 * \operatorname{sum}\left((-1)^{\wedge} n^{*} \operatorname{product}(8 * \mathrm{n}-\mathrm{j}, \mathrm{j}=0 . .2)^{*}(2 * \mathrm{n}+5)^{\wedge} 4 /(\right.\right.$ $2 * n) ! *{ }^{\wedge}(8 * n-3) * \sin (2 * n+5), n=0$..infinity), 14$)$;

$$
2.7815111865654 \cdot 10^{18}+0 . \mathrm{I}
$$

The imaginary part of the above answer obtained by Maple is 0 , so can be ignored.

\section{Conclusion}

From the above discussion, we know that Theorems 1, 2, 3 , and 4 are the theoretical basis for solving the partial differential problems we explored. And we see that differentiation term by term theorem occupies the pivotal position in our theoretical derivation. In fact, the application of this theorem is very extensive, and use it many difficult problems can be solved, we will continue to publish papers in this regard. On the other hand, it can be seen that Maple plays an important role in the auxiliary problem solving, we can even use Maple to design some types of partial differential problems, and try to find the key to solve them. In the future, we will study other calculus and engineering mathematics problems, and take these results as good materials for calculus and engineering mathematics on research and teaching.

\section{References}

[1] C. H., Bischof, G. Corliss, and A. Griewank, "Structured second and higher-order derivatives through univariate Tay- 
lor series," Optimization Methods and Software, 2, pp. 211-232, 1993.

[2] M. Hardy, "Combinatorics of partial derivatives," The Electronic Journal of Combinatorics 13, \#R1, 2006.

[3] L. E. Fraenkel, "Formulae for high derivatives of composite functions," Mathematical Proceedings of the Cambridge Philosophical Society, 83 : pp. 159-165, 1978.

[4] T-W, Ma, "Higher chain formula proved by combinatorics," The Electronic Journal of Combinatorics 16, \#N21, 2009.

[5] D. N. Richard, "An efficient method for the numerical evaluation of partial derivatives of arbitrary order," ACM Transactions on Mathematical Software (TOMS), 18(2), pp.
$159-173,1992$.

[6] A. Griewank and A. Walther, Evaluating Derivatives : Principles and Techniques of Algorithmic Differentiation, 2nd ed., SIAM, 2008.

[7] P. Franklin, Methods of Advanced Calculus, McGraw-Hill Co., Inc., chap. II, 1944.

[8] L. Flatto, Advanced Calculus, The Williams \& Wilkins Co., chap. 9, 1976.

[9] D. V. Widder, Advanced Calculus, 2 nd ed., Prentice-Hall, Inc, chap. $1 \& 4 ., 1961$.

[10] T. M. Apostol, Mathematical Analysis, 2nd ed., Addison-Wesley Publishing Co., Inc., p230, 1975. 